\title{
Anticancer compound Oplopantriol A kills cancer cells through inducing ER stress and BH3 proteins Bim and Noxa
}

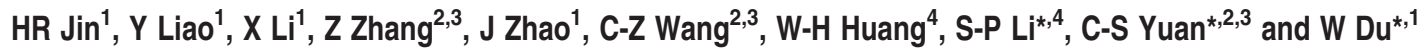

Oplopantriol-A (OPT) is a natural polyyne from Oplopanax horridus. We show here that OPT preferentially kills cancer cells and inhibits tumor growth. We demonstrate that OPT-induced cancer cell death is mediated by excessive endoplasmic reticulum (ER) stress. Decreasing the level of ER stress either by inactivating components of the unfolded protein response (UPR) pathway or by expression of ER chaperone protein glucose-regulated protein 78 (GRP78) decreases OPT-induced cell death. We show that OPT induces the accumulation of ubiquitinated proteins and the stabilization of unstable proteins, suggesting that OPT functions, at least in part, through interfering with the ubiquitin/proteasome pathway. In support of this, inhibition of protein synthesis significantly decreased the accumulation of ubiquitinated proteins, which is correlated with significantly decreased OPT-induced ER stress and cell death. Finally, we show that OPT treatment significantly induced the expression of BH3-only proteins, Noxa and Bim. Knockdown of both Noxa and Bim significantly blocked OPT-induced cell death. Taken together, our results suggest that OPT is a potential new anticancer agent that induces cancer cell death through inducing ER stress and BH3 proteins Noxa and Bim.

Cell Death and Disease (2014) 5, e1190; doi:10.1038/cddis.2014.169; published online 24 April 2014

Subject Category: Cancer

Due to rapid metabolism and growth rate, cancer cells generally exhibit hallmarks of increased cellular stresses including DNA damage/replication stress, proteotoxic stress, mitotic stress, metabolic stress, and oxidative stress. ${ }^{1}$ Therefore, cancer cells are often more dependent on the stress support pathways for survival, which can be exploited in cancer therapy by either stress sensitization or stress overload. ${ }^{1}$ In fact, a lot of currently approved chemotherapeutic drugs, such as Paclitaxel, 5-FU, or Bortezomib, kill cancer cells by inducing excessive cellular stress.

The endoplasmic reticulum (ER) is an essential intracellular organelle with multiple roles including lipid biosynthesis, intracellular membrane homeostasis, and promoting proper folding and modification of secreted and transmembrane proteins. Due to rapid glucose metabolism and growth rate, many cancer cells experience nutrient deprivation and hypoxia, both of which lead to the accumulation of unfolded proteins in the ER, resulting in ER stress which triggers the activation of the unfolded protein response (UPR).,3 There are three distinct branches of the UPR: PKR-like endoplasmic reticulum kinase (PERK), ATF6, and IRE1. 2,3 Activation of IRE1 catalyzes the splicing of the mRNA encoding X-box binding protein 1 (XBP1), a transcription factor that controls the transcription of genes involved in ER chaperones, ERassociated protein degradation (ERAD) genes, genes involved in autophagy, as well as genes involved in apoptosis. Activation of PERK leads to the phosphorylation of elF2 $\alpha$, which inhibits global translation of mRNAs and reduces the influx of new proteins into the ER to mitigate the burden on the ER protein folding machinery. However, PERK activation can also promote the translation of a subset of mRNAs, such as ATF4, which induce the expression of genes such as C/EBPhomologous protein (CHOP) and induce apoptosis. Therefore, UPR can promote survival by re-establishing ER homeostasis but excessive or prolonged ER stress will promote cell death.

A major target of the UPR that promotes survival and reestablishing of ER homeostasis is the ER chaperone protein glucose-regulated protein 78 (GRP78/BiP), which is required for protein folding and assembly, targeting misfolded proteins for degradation, $\mathrm{ER} \mathrm{Ca}^{2+}$ binding and controlling the activation of transmembrane ER stress receptors. ${ }^{4,5}$ Accumulating evidence has also demonstrated that overexpression of GRP78 is prominent in a wide variety of tumors

\footnotetext{
${ }^{1}$ Ben May Department for Cancer Research, The University of Chicago, Chicago, IL, USA; ${ }^{2}$ Tang Center for Herbal Medicine Research, The University of Chicago, Chicago, IL, USA; ${ }^{3}$ Department of Anesthesia and Critical Care, The University of Chicago, Chicago, IL, USA and ${ }^{4}$ State Key Laboratory of Quality Research in Chinese Medicine, Institute of Chinese Medical Sciences, University of Macau, Macao, China

${ }^{*}$ Corresponding authors: W Du, Ben May Department for Cancer Research, University of Chicago, Chicago, IL 60637, USA. Tel: +1 773 834 1949; Fax: +1 773702 4476; E-mail: wei@uchicago.edu

or C-S Yuan, Tang Center for Herbal Medicine Research, The University of Chicago, Chicago, IL 60637, USA. Tel: +1 773 702 1916; Fax: +1 773 834 0601 ; E-mail: cyuan@dacc.uchicago.edu

or S-P Li, State Key Laboratory of Quality Research in Chinese Medicine, Institute of Chinese Medical Sciences, University of Macau, Macao, China. Tel: +853 8397 4692; Fax: +853 2884 1358; E-mail: spli@umac.mo

Keywords: ER stress; Oplopantriol-A; unfolded protein response; noxa; bim

Abbreviations: ER, endoplasmic reticulum; UPR, unfolded protein response; GRP78, glucose-regulated protein 78; XBP1, X-box binding protein 1; CHOP, C/EBPhomologous protein; PERK, PKR-like endoplasmic reticulum kinase; ERAD, ER-associated protein degradation; LC3, Microtubule-associated protein light chain 3 Received 05.12.13; revised 28.1.14; accepted 30.1.14; Edited by A Stephanou
} 
and protects cancer cells against ER stress as well as a range of cancer therapeutic agents. ${ }^{5}$ On the other hand, terminally misfolded proteins that cannot be refolded by ER chaperone proteins are subject to ERAD, which involves their export back into the cytoplasm and subsequent degradation by the ubiquitin/proteasome pathway. ${ }^{6}$ Alternatively, they may be included into compacted aggresomes and recycled via autophagy. ${ }^{7}$ In fact, studies have shown that ER stress may lead to the activation of autophagy, and conversely, blockage of autophagy can enhance ER stress-induced cell death. ${ }^{8}$

If the function of the ER cannot be re-established by UPR, then excessive or sustained ER stress will induce cell death via IRE1-mediated activation of JNK signaling as well as through prolonged activation of the transcription factor CHOP. ${ }^{9}$ BCL2 family proteins are implicated in $\mathrm{CHOP}$ induced apoptosis and critically linked to cell life and death through the modulation of ER calcium homeostasis. ${ }^{10}$ Consistent with this, Bax- and Bak-deficient cells are shown to be resistant to ER stress-induced apoptosis ${ }^{11}$ and a number of proapoptotic BH3-only proteins, including Noxa, Bim, and BIK have been shown to be upregulated and required to mediate ER stress-induced cell death. ${ }^{12-16}$

Oplopantriol-A (OPT) is a novel natural polyyne isolated from Oplopanax horridus, a plant used extensively by indigenous people to heal a variety of ailments. ${ }^{17,18}$ OPT has been shown to inhibit cancer cell proliferation but the mechanisms involved are not known. ${ }^{19}$ In this report, we show that OPT preferentially kills cancer cells, and the cancer cell killing effect of OPT was mediated by the induction of ER

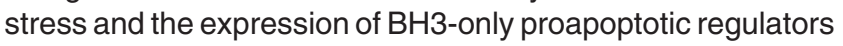
Noxa and Bim.

\section{Results}

OPT preferentially induces apoptosis in cancer cells. To investigate whether OPT can preferentially inhibit cell proliferation and induce cell death in cancer cells, we compared the effect of OPT on MDA-MB231 breast cancer and MCF10A untransformed human breast epithelial cells. The viability of cells treated with different concentrations of OPT was measured by MTT (3-(4,5-dimethylthiazol-2-yl)2,5-diphenyltetrazolium bromide) assay. As shown in Figure 1a, a significant portion of the MDA-MB231 cells lost viability with $3 \mu \mathrm{M}$ OPT treatment while a majority of MCF10A cells survived at much higher concentrations of OPT $(40 \mu \mathrm{M})$. To further characterize the effect of OPT, we stained MCF10A and MDA-MB231 cells with Annexin-V and PI. OPT treatment significantly increased Annexin-V and $\mathrm{PI}$ staining of the MDA-MB231 but not the MCF10A cells (Figures $1 \mathrm{~b}$ and $\mathrm{c}$ ). Additionally, OPT preferentially killed MLL-AF9 mouse leukemia cells but not the mouse bone marrow cells (Figure 1e) and induced a significant level of cell death in HCT116 colorectal cancer cells as well as in KOPN1 lymphoblastic leukemia cells (Figures 1d and f). These data indicate that OPT preferentially kills cancer cells. We used a caspase-3 activity indicator (C3AI), which shows a
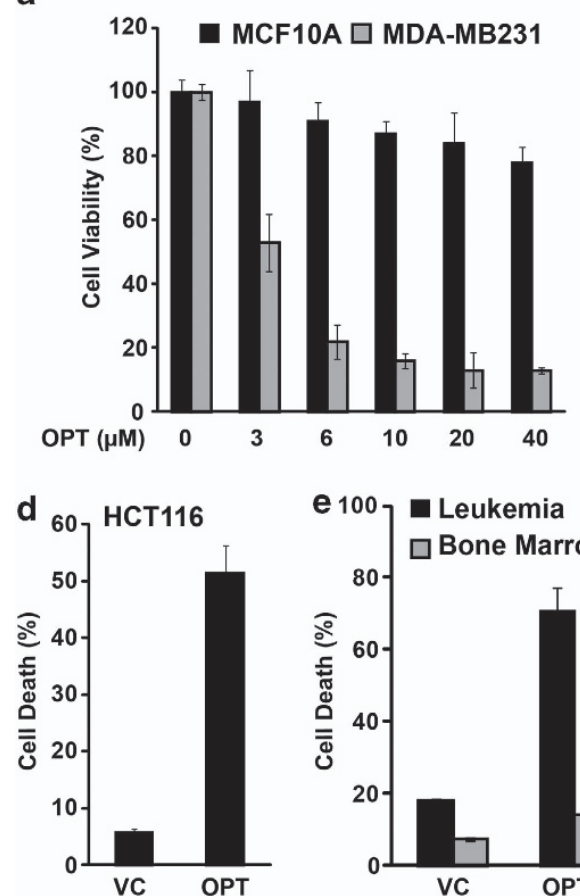

b
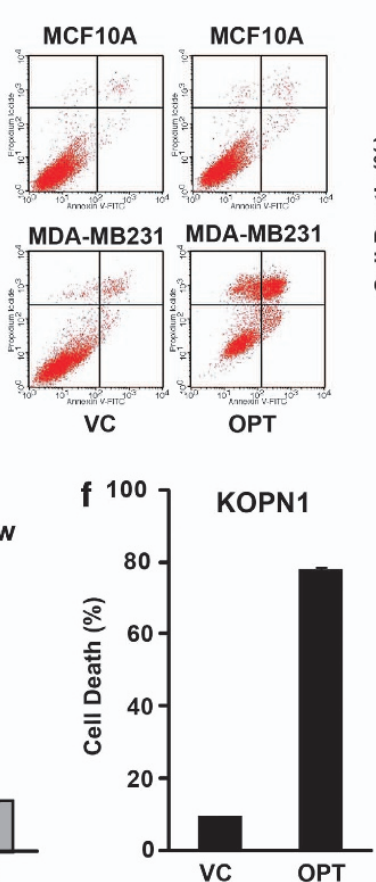
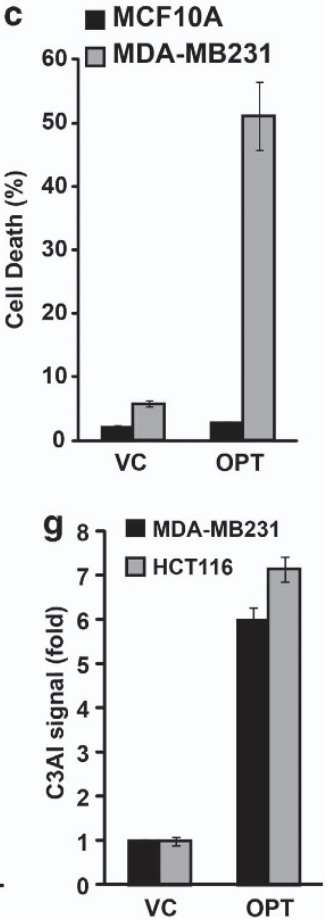

Figure 1 OPT preferentially induces cancer cell death. (a) Untransformed human epithelial cells MCF10A and the breast cancer cells MDA-MB231 were treated with different concentrations of OPT and cell viability at $48 \mathrm{~h}$ after treatment was determined by MTT assay. (b and $\mathbf{c}$ ) MCF10A and MDA-MB231 cells were treated with OPT $(10 \mu \mathrm{M})$ for $48 \mathrm{~h}$, and cell death was quantified by FACS after staining with Annexin V-FITC and propidium iodide. (d) HCT116 cells were treated with OPT (10 $\mu \mathrm{M})$ for $48 \mathrm{~h}$, and cell death was quantified by FACS. (e) Mouse bone marrow cells and MLL-AF9 mouse leukemia cells were treated with OPT (1.5 $\mu \mathrm{M})$ for $48 \mathrm{~h}$, and apoptosis was quantified by FACS. (f) Human leukemia KOPN1 cells were treated with OPT (1.5 $\mu \mathrm{M})$ for $48 \mathrm{~h}$, and apoptosis was quantified by FACS. (g) C3Al transduced MDA-MB231 and HCT116 cells were treated with OPT $(10 \mu \mathrm{M})$ for $48 \mathrm{~h}$, and the level of fluorescence was quantified by FACS 
fluorescence only after cleavage by caspase-3 like proteases, ${ }^{20}$ to further characterize whether OPT-induced cell death is correlated with increased caspase-3 activity. Indeed, OPT treatment significantly increased C3Al fluorescence (Figure 1g). These observations suggest that OPT-induced cell death is mostly mediated by caspase-dependent apoptosis.

OPT inhibits tumor growth in xenograft tumor model. A xenograft model of HCT-116 human colorectal cancer cells was used to determine whether OPT can inhibit cancer growth in vivo. Exponentially growing firefly luciferase-tagged HCT-116 cells were inoculated into the flanks of athymic nude mice. Beginning on Day 1, animals were also administered with OPT or vehicle at $15 \mathrm{mg} / \mathrm{kg} / \mathrm{day}$. Tumor growth was measured by Xenogen bioluminescence imaging every week. Treatment with OPT significantly decreased the Xenogen imaging signal (Figure 2a). Quantitative analysis of the imaging data (Figure $2 \mathrm{~b}$ ) revealed that treatment with $15 \mathrm{mg} / \mathrm{kg}$ of OPT significantly inhibited xenograft tumor growth starting from week $3\left({ }^{*}\right.$ and ${ }^{* *}$ indicate $P<0.05$ or $P<0.01$, respectively). These results demonstrate that OPT can inhibit human tumor growth in a xenograft model.

OPT-induced cell death is correlated with ER stress induction. To investigate the mechanisms by which OPT induces cancer cell death, we characterized the ability of OPT to induce ER stress, ROS, and autophagy. OPT treatment induced significant levels of XBP1 splicing, $\mathrm{CHOP}$ expression, as well as GRP78 protein accumulation in MDAMB231 cancer cells (Figures $3 a$ and b), and a similar effect was observed by the ER stress inducer Tunicamycin (Supplementary Figure S1). Interestingly, as shown in Figure 3b, the XBP1 splicing and CHOP mRNA induction was detectable as early as $2 \mathrm{~h}$ and persistently induced at $24 \mathrm{~h}$ after OPT treatment. However, GRP78 mRNA was not induced $2 \mathrm{~h}$ after OPT treatment (Supplementary Figure S2) and significantly increased GRP78 protein levels were observed after $8 \mathrm{~h}$ of OPT treatment (Figure 3a), significantly later than the induction of $\mathrm{CHOP}$ and XBP1 splicing. As CHOP functions to promote ER stress-induced cell death while GRP78 protects cells from ER stress-induced cell death, the early induction of proapoptotic genes such as $\mathrm{CHOP}$ and the relatively late induction of protective mechanisms such as GRP78 potentially contribute to the cell death induced by OPT treatment. To determine whether ER stress induction correlates with OPT-induced cell death, we determined the level of XBP1 splicing in the OPTsensitive as well as resistant cells. MDA-MB231 and HCT116 cancer cells, which are sensitive to OPT treatment, exhibited a high level of XBP1 splicing after OPT treatment while MCF10A cells, which are resistant to OPT treatment, did not exhibit OPT-induced XBP1 splicing (Figures $3 \mathrm{c}$ and $\mathrm{d}$ ). Therefore, the ability of OPT to induce ER stress correlates nicely with OPT-induced cell death.

We also investigated the ability of OPT to induce oxidative stress and autophagy in cancer cells. Interestingly, OPT
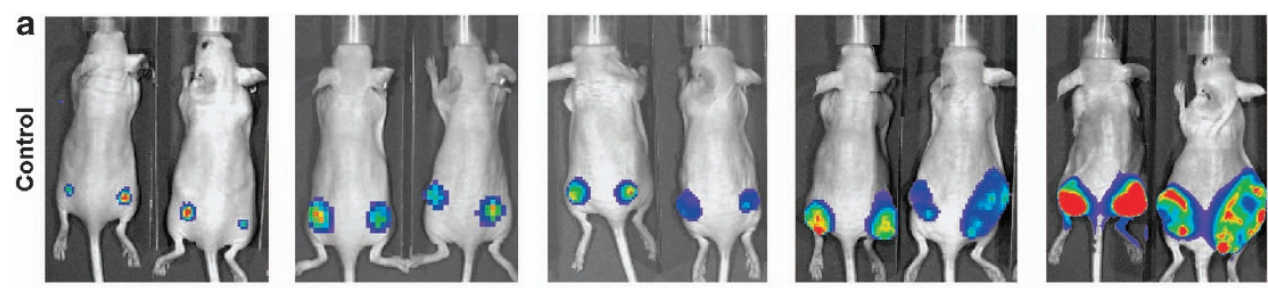

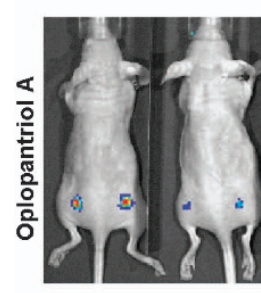

Week 1

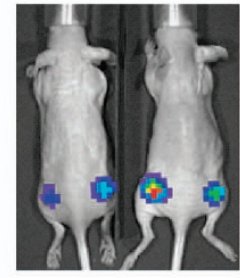

Week 2

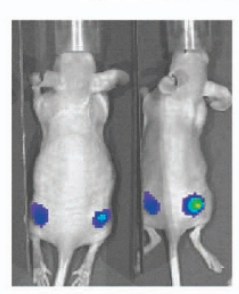

Week 3

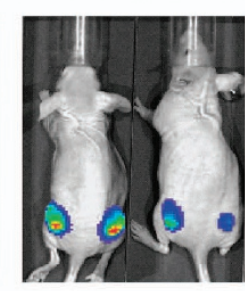

Week 4

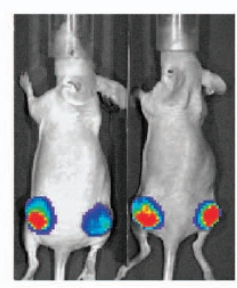

Week 5

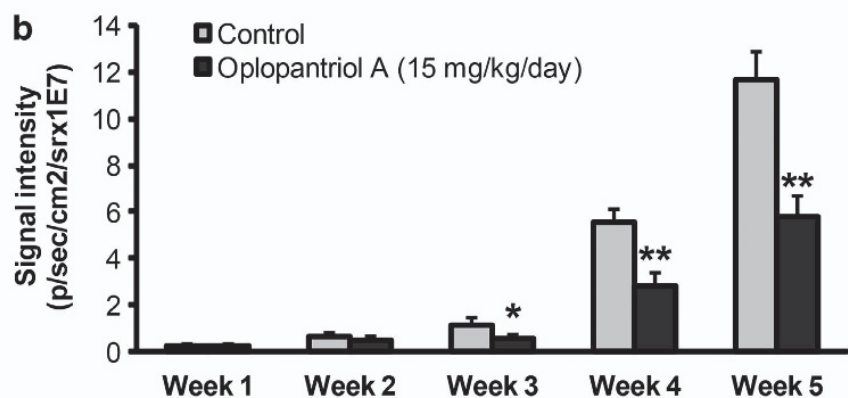

Figure 2 In vivo antitumor evaluation of Oplopantriol A using a xenograft model. (a) Firefly luciferase-tagged HCT-116 cells were injected into both flanks of athymic mice subcutaneously ( $n=10 /$ group), and the tumor sizes after treatment with solvent control or $15 \mathrm{mg} / \mathrm{kg} / \mathrm{day}$ of Oplopantriol A were measured on a weekly basis by xenogen bioluminescence imaging. Representative xenogen imaging results are shown. (b) Quantitative analysis of xenogen bioluminescence imaging. Average tumor sizes at the indicated time points are represented with imaging signal intensities (in photons $/ \mathrm{second} / \mathrm{cm}^{2} /$ steradian) as mean \pm standard error. ${ }^{*} P<0.05,{ }^{* \star} P<0.01$ versus control 


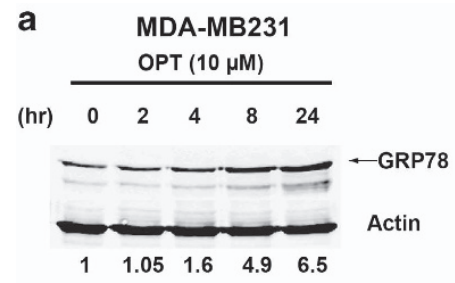

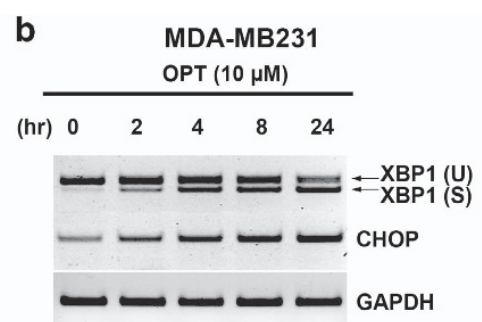

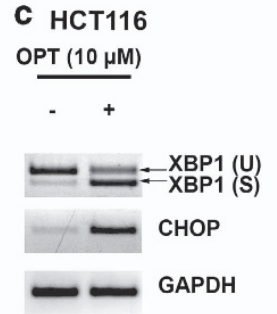

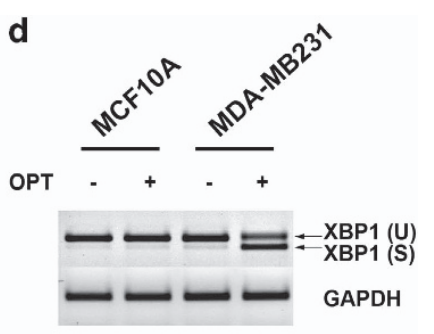

h
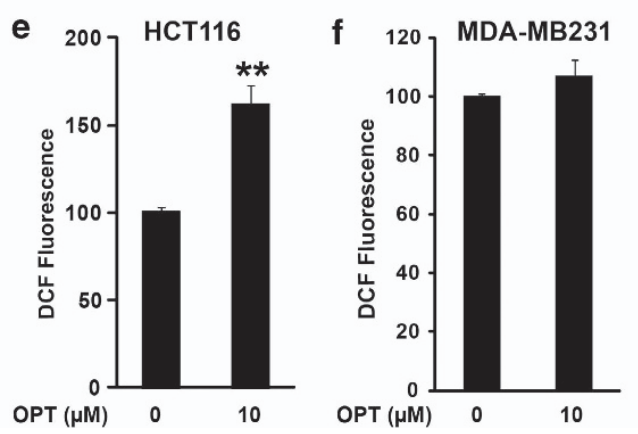

g

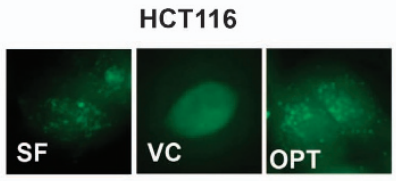

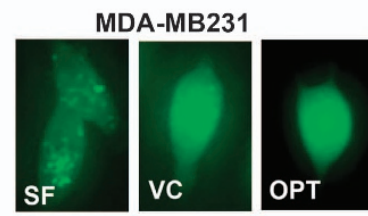

Figure 3 The effects of OPT on endoplasmic reticulum stress, ROS, and autophagy. (a) Western blot showing the induction of GRP78 in MDA-MB231 cells treated with OPT at different time points. Full-length GRP78 protein is indicated by an arrow. The numbers indicate the normalized level of GRP78. (b) RT-PCR analysis showing the induction of XBP1 splicing and CHOP expression after MDA-MB231 cells were treated with OPT at different time points. (c) RT-PCR analysis showing the induction of XBP1 splicing and CHOP expression after HCT116 cells were treated with OPT for $8 \mathrm{~h}$. (d) MCF10A and MDA-MB231 cells were treated with OPT for $8 \mathrm{~h}$, and the XBP1 splicing and CHOP expression were detected by RT-PCR. (e) HCT116 cells were treated with OPT for $16 \mathrm{~h}$, and the ROS level was determined. (f) MDA-MB231 cells were treated with OPT for $16 \mathrm{~h}$, and the ROS level was determined. ( $\mathrm{g}$ and $\mathbf{h}$ ) HCT116 and MDA-MB231 cells expressing EGFP-LC3 were treated with vehicle control (VC) or $6 \mu \mathrm{M}$ OPT for $30 \mathrm{~h}$ and were imaged with a fluorescence microscope. Serum-free (SF) treatment was used as a positive control for autophagy. ${ }^{* *}$ indicates $P<0.01$

treatment induced significantly increased ROS levels in HCT116 colorectal cancer cells (Figure 3e) but not in MDAMB231 cells (Figure 3f), suggesting that OPT-induced ROS production is cell type dependent. Similarly, OPT induced a significant Microtubule-associated protein light chain 3 (LC3)GFP puncta formation in HCT116 but not in MDA-MB231 cells (Figures $3 \mathrm{~g}$ and $\mathrm{h}$ ), indicating OPT induced autophagy in HCT116 but not in MDA-MB231 cells. Therefore, OPT can induce ROS and autophagy only in a subset of OPT-sensitive cancer cells.

ER stress contributes to OPT-induced apoptosis. Since OPT-induced ER stress is correlated with cell death induction in all the cells examined, we further characterized the contribution of ER stress to OPT-induced cell death. ER chaperone protein GRP78 is an important regulator of ER stress. Overexpression of ER chaperone protein GRP78 using a myc-tagged GRP78 construct (Figure 4b) significantly decreased OPT-induced ER stress as shown by decreased levels of XBP1 splicing (Figure 4c) and decreased OPT-induced cell death in MDA-MB231 cells (Figure 4a). In contrast, knockdown of GRP78 using shRNA constructs against GRP78 decreased GRP78 levels (Figure 4e), increased ER stress (Figure 4f), and significantly increased OPT-induced cell death (Figure 4d).

To further confirm that ER stress contributes to OPTinduced cell death, we determined the effect of knocking out components of the UPR pathway, PERK and XBP1, on OPTinduced cell death using established mouse embryonic fibroblast (MEF) cells. $^{21} \mathrm{XBP}^{-1-}$ MEFs showed an increased basal level of XBP1 splicing, but significantly reduced OPT-induced ER stress, as shown by reduced induction of GRP78 expression and XBP1 splicing (Figures $4 \mathrm{j}$ and $\mathrm{k}$ ). The reduced $\mathrm{ER}$ stress induction is correlated with reduced OPT-induced cell death in $\mathrm{XBP}^{-1-} \mathrm{MEFs}$ (Figure 4i). Similarly, PERK ${ }^{-1-}$ MEFs also significantly decreased OPT-induced ER stress as shown by reduced GFP78 induction (Figure 4h), which correlated with decreased OPT-induced cell death (Figure $4 \mathrm{~g}$ ). Therefore, OPT-induced ER stress contributes to its induction of cell death.

Inhibition of protein synthesis will decrease protein influx to the ER, which can potentially help to restore ER homeostasis. Indeed, blocking protein synthesis can block the accumulation of unfolded/misfolded proteins in ER and therefore decrease ER stress and ER stress-induced cell death. ${ }^{21}$ To further characterize the molecular mechanism of OPT-induced ER stress and cell death, we tested the effect of blocking protein synthesis by cycloheximide $(\mathrm{CHX})$. Pre-treatment with $\mathrm{CHX}$ reduced OPT-induced ER stress and inhibited the induction of CHOP in both MDA-MB231 cells and HCT116 cells (Figures $5 a$ and b). The reduced ER stress and CHOP induction correlated with significantly inhibited OPT-induced cell death (Figures $5 c$ and $d$ ) in these cancer cells. These data further confirm that OPT-induced cell death is associated with its induction of ER stress.

OPT induces accumulation of ubiquitinated proteins in cancer cells. ERAD has an important role in maintaining the homeostasis of ER through delivering of misfolded protein to the cytosol for degradation by the proteasome-ubiquitin pathway. Interestingly, OPT treatment induced significantly increased accumulation of ubiquitinated proteins in both 
a
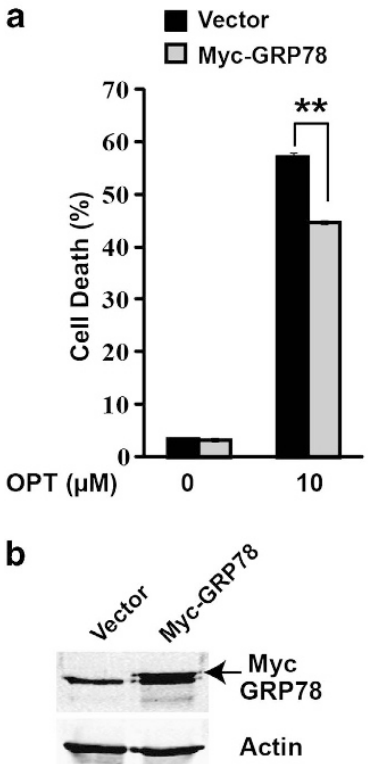

d

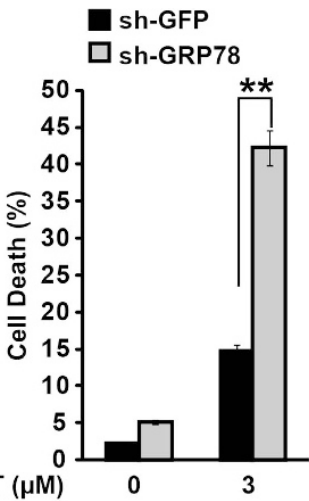

e

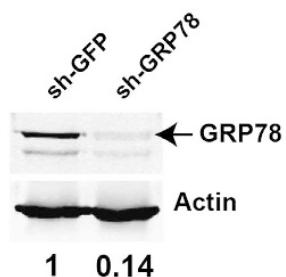

g

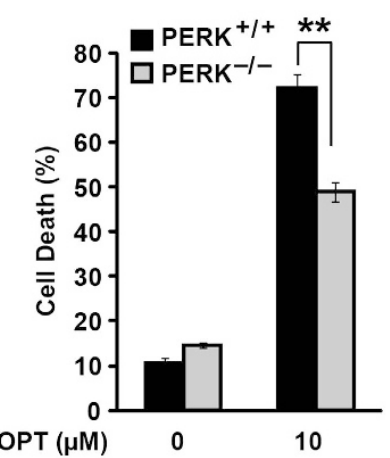

h

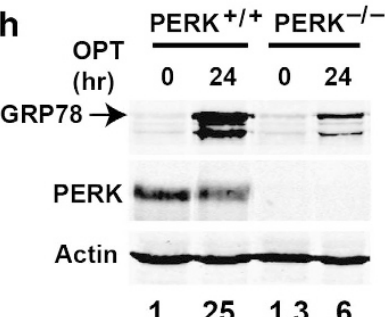

$\begin{array}{llll}1 & 25 & 1.3 & 6\end{array}$ i

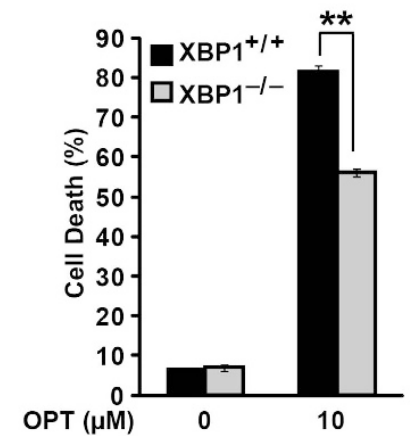

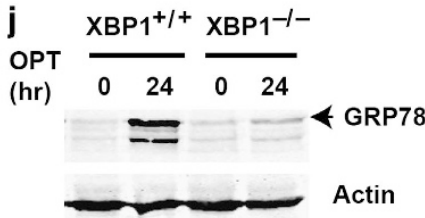

$\begin{array}{llll}1 & 8.7 & 2.0 & 3.0\end{array}$
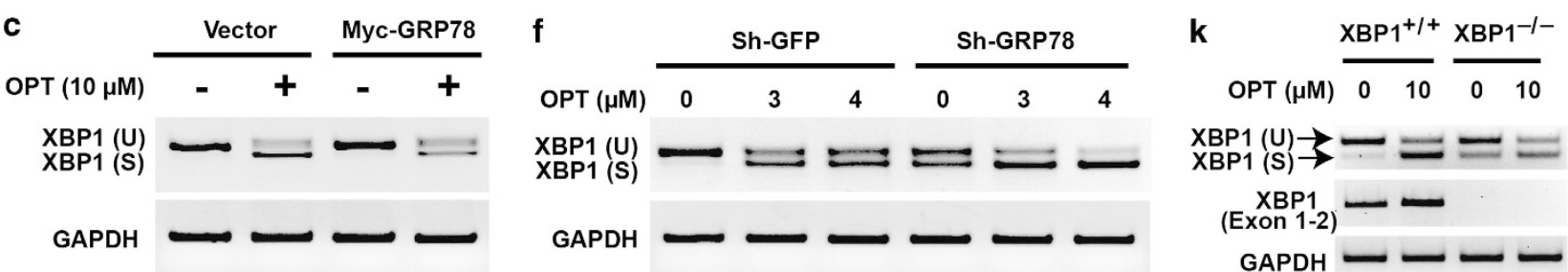

Figure 4 ER stress contributes to OPT-induced cancer cell death. (a) MDA-MB231 cells infected with lentivirus expressing either myc-GRP78 or empty vector were treated with different concentrations of OPT for $48 \mathrm{~h}$, and cell death was determined. (b) Extracts from vector control or myc-GRP78-expressing cells were analyzed by western blotting. Anti-Myc antibody was used to detect myc-GRP78 (indicated by an arrow). The blot was re-probed with an anti GRP78 antibody, which detects the endogenous GRP78 bands (below myc-GRP78). (c) RT-PCR analysis of the induction of XBP1 splicing after myc-GRP78 or vector control-infected MDA-MB231 cells were treated with $10 \mu \mathrm{M}$ OPT for $8 \mathrm{~h}$. (d) MDA-MB231 cells infected with sh-GRP78 constructs or sh-GFP control were treated with $3 \mu \mathrm{M}$ OPT for $48 \mathrm{~h}$, and cell death was determined. (e) Extracts from sh-GFP or sh-GRP78 cells were analyzed by western blots to determine the levels of GRP78 protein (indicated by an arrow). (f) RT-PCR analysis of the induction of XBP1 splicing after sh-GRP78 or sh-GFP infected MDA-MB231 cells were treated with 3 and $4 \mu \mathrm{M}$ OPT for $8 \mathrm{~h}$. (g and i) Wild-type and PERK ${ }^{-1-}$ or XBP1 ${ }^{-1-}$ mouse embryonic fibroblasts were treated with $10 \mu \mathrm{M}$ OPT for $48 \mathrm{~h}$, and cell death was determined. (h and j) Wild-type and PERK ${ }^{-1-}$ or XBP1 ${ }^{-1-}$ mouse embryonic fibroblasts were treated with $10 \mu \mathrm{M}$ OPT for the indicated periods, and then GRP78 and PERK expression levels were determined by western blot analysis. The numbers in (e, $\mathbf{h}$, and j) indicate the normalized level of GRP78. (k) Wild-type and XBP1 ${ }^{-1-}$ mouse embryonic fibroblasts were treated with $10 \mu \mathrm{M}$ OPT for $8 \mathrm{~h}$, and the XBP1 splicing and XBP1 (Exons 1-2) expression were detected by RT-PCR. Note: XBP1 knockout does not remove the region that is used to assay alternative splicing; therefore, XBP1 splicing can still be detected in the XBP1 knockout MEF and used as a readout for ER stress. ${ }^{* *}$ indicates $P<0.01$

MDA-MB231 and HCT116 cancer cells (Figures 5e-g) but not in untransformed MCF10A breast epithelial cells (Figure $5 \mathrm{e}$ ). The accumulation of ubiquitinated protein correlated with the ability of OPT to induce cell death and ER stress (Figures $1 c, d, 3 c$ and $d$ ). Furthermore, the inhibition of ER stress and cell death by $\mathrm{CHX}$ correlated with significantly decreased accumulation of ubiquitinated proteins (Figures $5 a-d, f$ and $g$ ). These observations suggest that OPT may interfere with the degradation of ubiquitinated proteins, causing the accumulation of misfolded proteins resulting in the induction of ER stress.

To further test the possibility that OPT may interfere the ubiquitin/proteasome-mediated protein degradation, we determined the effect of OPT on c-Myc protein, which is an unstable protein degraded by the ubiquitin/proteasome pathway. Proteasome inhibitor MG132 treatment was used as a positive control, and led to increased c-Myc protein accumulation (Figure 5h). Interestingly, OPT treatment induced similar accumulation of c-Myc protein in a time-dependent manner (Figure 5h). These results further support the hypothesis that OPT interferes with the ubiquitin/proteasome pathway.

Effect of OPT-induced ROS and autophagy on cell death induction. In addition to ER stress, OPT also induced ROS and autophagy in HCT116 cells (Figures $3 \mathrm{e}-\mathrm{g}$ ). To investigate whether OPT-induced ROS and autophagy affect OPTinduced cell death, we determined the effect of antioxidants, $\mathrm{N}$-acetyl cysteine (NAC), and autophagy inhibitor Chloroquine (CQ). NAC treatment partially decreased OPT-induced cell death in HCT116 cells (Supplementary Figure S3a), suggesting that OPT-induced ROS contributes to cell death induction in HCT116 cells. In contrast, inhibition of autophagy 


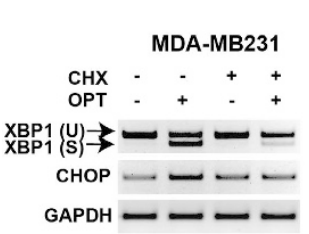

b

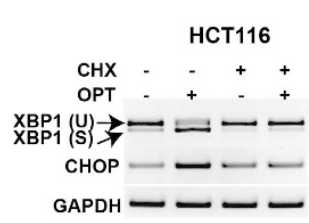

C

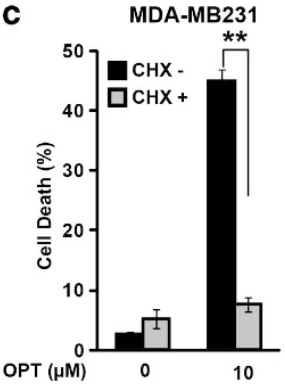

d HCT116

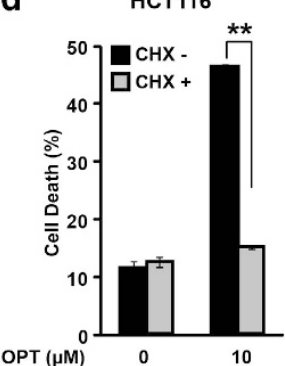

e

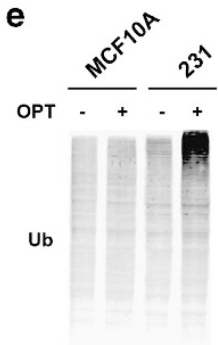

f

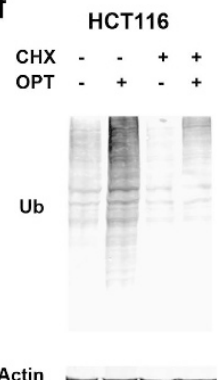

g CHX - - + +

OPT -+++

Ub

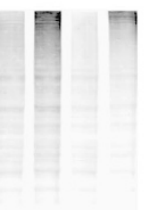

h

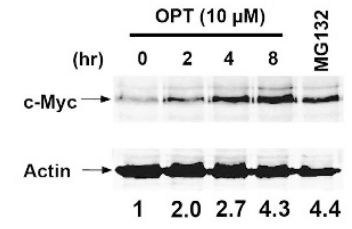

Actin

Actin $\longrightarrow \longrightarrow$

Figure 5 OPT-induced ER stress is correlated with an accumulation of ubiquitinated proteins. (a and $\mathbf{b}$ ) MDA-MB231 and HCT116 cells were treated with $10 \mu \mathrm{M}$ OPT for $8 \mathrm{~h}$ with or without cycloheximide $(10 \mu \mathrm{g} / \mathrm{ml})$, and XBP1 splicing and CHOP expression were determined by RT-PCR. (c and d) MDA-MB231 and HCT116 cells were treated with $10 \mu \mathrm{M}$ OPT for $48 \mathrm{~h}$ with or without cycloheximide $(10 \mu \mathrm{g} / \mathrm{ml})$, and then cell death was determined. (e) MCF10A and MDA-MB231 cells were treated with $10 \mu \mathrm{M}$ OPT for $8 \mathrm{~h}$, and then the level of ubiquitinated proteins was determined by western blot analysis. (f and $\mathbf{g}$ ) HCT116 and MDA-MB231 cells were treated with $10 \mu \mathrm{M}$ OPT for $8 \mathrm{~h}$ with or without cycloheximide $(10 \mu \mathrm{g} / \mathrm{ml})$, and the ubiquitinated protein expression level was determined by western blot analysis. (h) MDA-MB231 cells were treated with $10 \mu \mathrm{M}$ OPT in a time-dependent manner or with $20 \mu \mathrm{M}$ of the proteasome inhibitor MG132 for $8 \mathrm{~h}$, and the level of c-Myc protein was determined by western blot analysis. The numbers indicate the normalized c-Myc level. ${ }^{* *}$ indicates $P<0.01$

by $C Q$ significantly increased cell death in HCT116 cells (Supplementary Figure S3c), indicating that OPT-induced autophagy protects HCT116 cells from apoptosis induction. As expected, neither NAC nor CQ affects OPT-induced cell death in MDA-MB231 cells (Supplementary Figures S3b and d), which do not show ROS or autophagy induction by OPT (Supplementary Figures $3 f$ and $h$ ).

Noxa and Bim have an important role during OPTinduced cell death. Activation of the proapoptotic proteins, $B A X$ or $B A K$, is required for cell death in response to diverse stimuli, including ER stress. ${ }^{11}$ Activation of BAX and BAK proteins is controlled by the $\mathrm{BH} 3$-only proapoptotic proteins, which are induced by diverse stimuli to induce apoptosis. ${ }^{22}$ Indeed previous studies demonstrated that BH3-only proteins have an important role during ER stress and proteasome inhibitor-mediated cell death. ${ }^{12-16}$ To determine whether apoptotic regulators contribute to OPT-induced cell death, we investigated the expression of BH3-only proteins that are induced by OPT. We found the levels of apoptotic regulators Bim and Noxa to be significantly induced by OPT treatment as determined by qRT-PCR in both MDA-MB231 and HCT116 cells (Figures 6a-d). The induction of Bim by OPT can also be detected at the protein level (Figures $6 i$ and j). To further investigate whether the observed Bim and Noxa induction contributes to OPT-induced cell death, we used lentivirus to introduce shRNA to specifically knock down Noxa or Bim expression. Knockdown of Bim significantly decreased OPT-induced Bim levels (Figure 6i) and significantly decreased OPT-induced cell death in both MDA-
MB231 (Figure 6e) and HCT116 cells (Figure 6h). Similarly, knockdown of Noxa significantly blocked OPT-induced Noxa expression (Figure 6k) and significantly reduced OPTinduced cell death in both MDA-MB231 and HCT116 cells (Figures $6 f$ and g). Furthermore, knockdown of both Noxa and Bim largely blocks OPT-induced cell death (Figure 6l). Therefore, both Noxa and Bim contribute to OPT-induced cancer cell death.

MCL1 is an unstable protein with a half-life of around $40 \mathrm{~min}^{23}$ Interestingly, in addition to inducing the expression of proapoptotic regulators Noxa and Bim, OPT also induced accumulation of the antiapoptotic MCL1 protein (Figure 7a). The effect of OPT on MCL1 is likely due to its ability to block protein degradation since the MCL1 mRNA was not significantly affected by OPT treatment (Supplementary Figure S4) and proteasome inhibitor MG132 also induced MCL1 accumulation (Figure 7a). Therefore, OPT-induced cell death is likely determined by the relative increase in the activities of the proapoptotic Noxa and Bim versus the antiapoptotic MCL1. Consistent with this idea, knockdown of MCL1 (Figure $7 b$ ) significantly increased OPT-induced cell death in MDA-MB231 and HCT116 cancer cells (Figures 7c and d). These observations suggest that the anticancer effects of OPT can potentially be enhanced by simultaneously inhibiting MCL1.

\section{Discussion}

Our results show that OPT preferentially kills cancer cells and inhibits tumor growth through inducing excessive ER stress. 
a

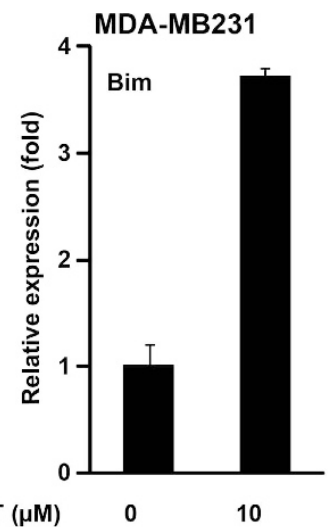

e

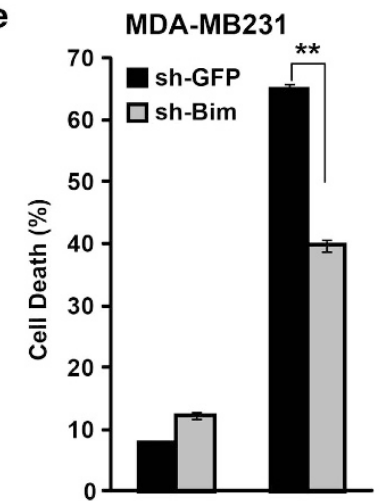

$\begin{array}{lll}\text { OPT }(\mu M) & 0 & 10\end{array}$

i

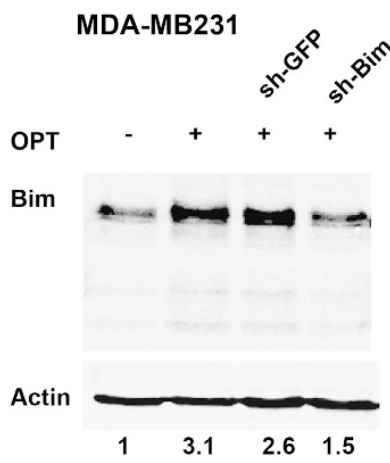

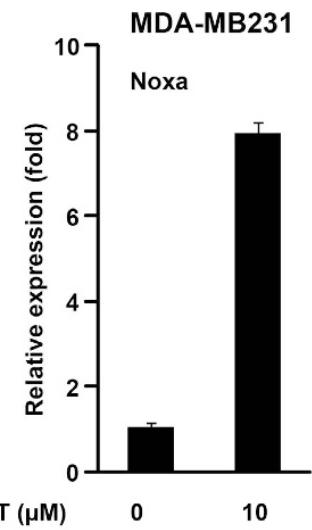

c

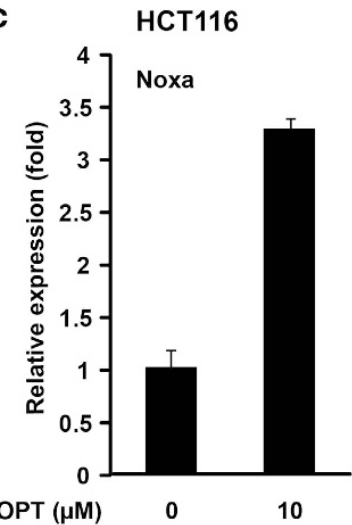

d

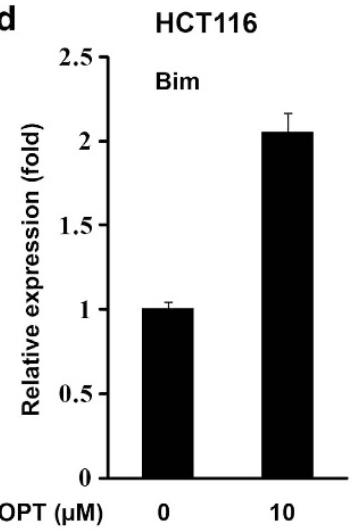

f

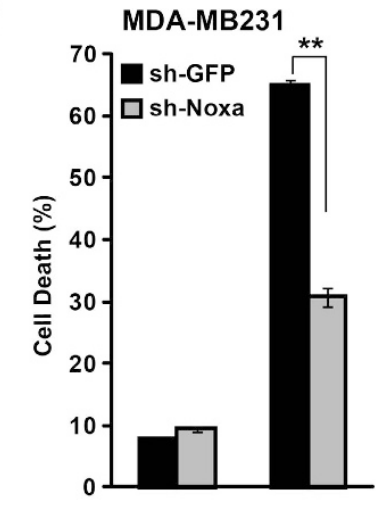

$\begin{array}{lll}\text { OPT }(\mu \mathrm{M}) & 0 & 10\end{array}$

j

k
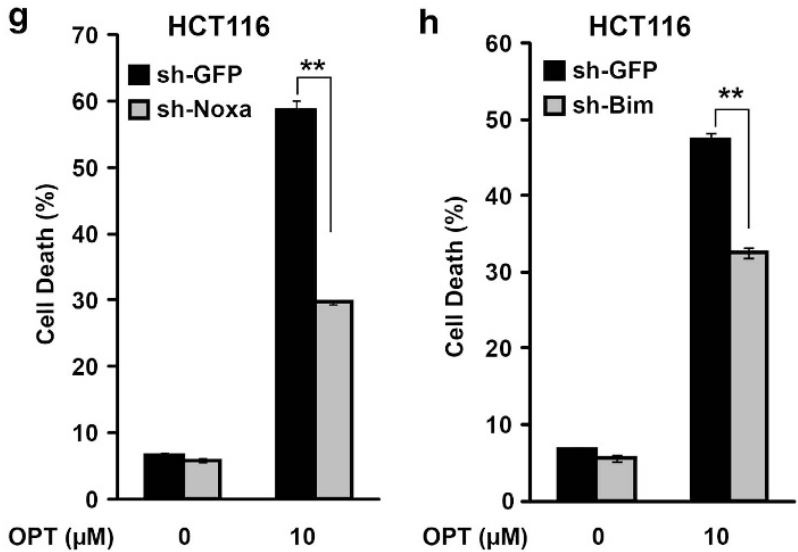

I

MDA-MB231

sh-GFP+sh-GFP

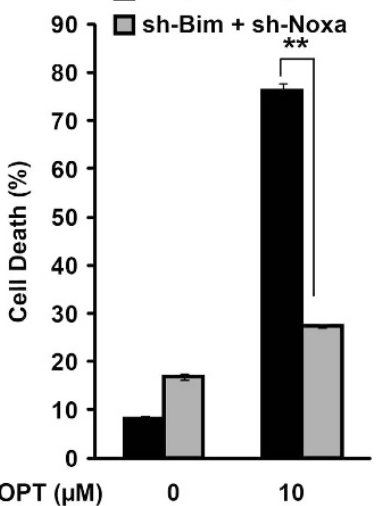

Figure 6 BH3-only proteins Noxa and Bim mediate OPT-induced cell death. (a-d) MDA-MB231 (a and $\mathbf{b}$ ) and HCT116 (c and $\mathbf{d}$ ) cells were treated with OPT for $24 \mathrm{~h}$, and then the expression levels of Bim ( $\mathbf{a}$ and $\mathbf{d})$ and Noxa ( $\mathbf{b}$ and $\mathbf{c}$ ) were determined by qRT-PCR. (e-h) MDA-MB231 cells (e and $\mathbf{f}$ ) and HCT116 cells ( $\mathbf{g}$ and $\mathbf{h}$ ) were infected with sh-Bim constructs (e and $\mathbf{~}$ ), sh-Noxa (f and $\mathbf{g}$ ) or sh-GFP control, and were treated with $10 \mu \mathrm{M} \mathrm{OPT}$ for $48 \mathrm{~h}$, and cell death was determined. (i) Western blot showing the induction of Bim in MDA-MB231 cells treated with $10 \mu \mathrm{M}$ OPT for $24 \mathrm{~h}$, and also showing decreased OPT-induced Bim protein accumulation with sh-Bim treatment. (j) Western blot showing the induction of Bim in HCT116 cells treated with10 $\mu \mathrm{M}$ OPT for $24 \mathrm{~h}$. The numbers in (i and $\mathbf{j}$ ) indicate the normalized level of Bim. (k) RT-PCR showing knockdown of Noxa by sh-Noxa construct blocks OPT-induced Noxa expression. (I) MDA-MB231 cells were double infected with sh-Bim and sh-Noxa construct or sh-GFP and sh-GFP construct, and cell was treated with $10 \mu \mathrm{M}$ OPT for $48 \mathrm{~h}$, and cell death was determined. ${ }^{*}$ indicates $P<0.01$

Decreasing the level of ER stress by inactivating components of the UPR pathway or by overexpressing GRP78 significantly inhibited OPT-induced cell death. We show that OPT-induced ER stress is correlated with an accumulation of ubiquitinated protein and the stabilization of unstable proteins. These observations suggest that OPT functions, at least in part, by interfering with the ubiquitin/proteasome degradation pathway. In support of this idea, inhibition of protein synthesis decreased accumulation of ubiquitinated proteins, reduced OPT-induced ER stress, and significantly inhibited OPTinduced cell death. The ubiquitin/proteasome system regulates many cellular functions, including the level of key 
a

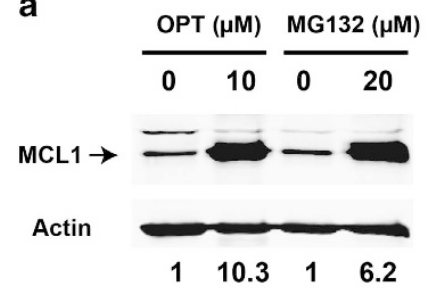

c

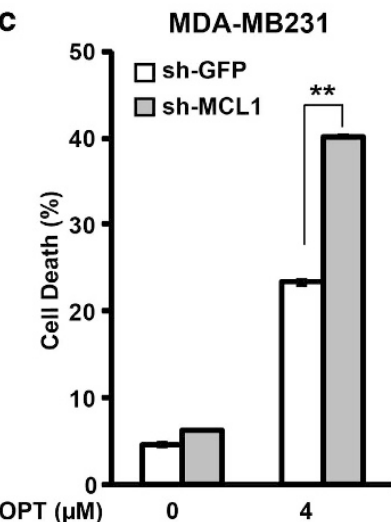

b HCT116 MDA-MB231

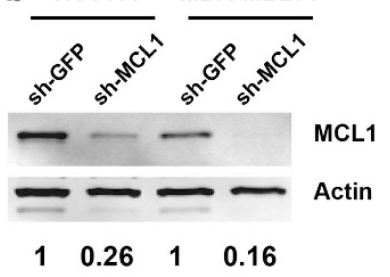

d

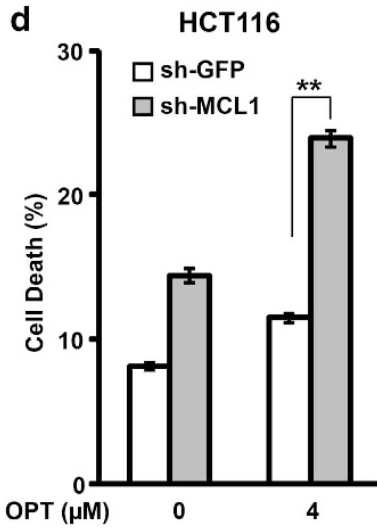

Figure 7 Knockdown of MCL1 sensitizes OPT-induced cell death. (a) MDAMB231 cells were treated with $10 \mu \mathrm{M}$ OPT or $20 \mu \mathrm{M} \mathrm{MG132}$ for $24 \mathrm{~h}$, and then the level of MCL1 protein was determined by western blot analysis. (b) Extracts from shGFP or sh-MCL1 cells were analyzed by western blots to determine the levels of $M C L 1$ protein. The numbers in ( $\mathbf{a}$ and $\mathbf{b})$ indicate the normalized level of MCL1. (c and d) MDA-MB231 and HCT116 cells infected with sh-MCL1 constructs or shGFP control were treated with $4 \mu \mathrm{M}$ OPT for $48 \mathrm{~h}$, and cell death was determined. ** Indicates $P<0.01$

regulators of the cell proliferation and survival systems, as well as removal of unfolded/misfolded proteins via ERAD, to maintain ER homeostasis. The ubiquitin/proteasome system is a potential target for cancer therapeutic agents. Indeed, proteasome inhibitor Bortezomib has been approved for treating multiple myeloma and mantle cell lymphoma. Although it is traditionally thought that the stabilization of a particular subset of proteins might be responsible for the antitumorigenic activity of bortezomib, it is equally likely that its efficacy results from enhanced proteotoxic stress.

The Bcl2 family of proteins includes the BH3-only type of proapoptotic regulators that are induced by different cell death signals, as well as the antiapoptotic regulators such as Bcl-2 and MCL1. The balance between the proapoptotic and the antiapoptotic regulators is critical in determining whether a cell will survive or die. We show that Bim and Noxa are induced by OPT and that knockdown of both Bim and Noxa significantly blocked OPT-induced cell death. Therefore, Bim and Noxa are critical mediators of OPT-induced cell death. These results are consistent with previous reports on the involvement of Bim and Noxa in mediating ER stress or proteasome inhibition-induced apoptosis. ${ }^{12-16}$ Interestingly, OPT treatment also significantly increased the level of MCL1, which is also a highly unstable protein. The observed MCL1 stabilization is likely related to the role of OPT in blocking protein degradation and potentially protects cells from undergoing apoptosis. Decreasing the level of MCL1 significantly sensitized cancer cells to OPT-induced cell death, suggesting that

OPT-induced cancer cell death can be further enhanced by inhibitors of MCL1.

Natural products are a valuable source of drugs for disease intervention and prevention. For example, current important anticancer drugs such as paclitaxel and vinblastine were initially discovered from natural products. Similarly, both salicylate, the active metabolite of aspirin, and the antidiabetic drug metformin are originally derived from herbal medicines and have been linked with reduced cancer incidence. Our demonstration that OPT preferentially kills cancer cells and inhibits tumor growth by interfering the ubiquitin/proteasome pathway and inducing the expression of Noxa and Bim suggest OPT can potentially be used to in cancer prevention or design new combinatorial therapies based on complementary mechanisms of action of different available anticancer drugs.

\section{Materials and Methods}

Plant materials. The root bark of $O$. horridus (Sm.) Miq., from Oregon, USA, was obtained from Pacific Botanicals (Grants Pass, OR, USA) and authenticated by Dr. Chong-Zhi Wang. The voucher specimens were deposited in the Tang Center for Herbal Medical Research at the University of Chicago.

Extraction, compound isolation, and structural identification. Air-dried, powdered root bark of $O$. horridus was extracted with $80 \%$ ethanol under refluxing, suspended in water, then extracted with petroleum ether $\left(60-90^{\circ} \mathrm{C}\right)$, ethyl acetate, and $n$-butanol successively to give three fractions. The ethyl acetate fraction was separated by silica gel, RP- $\mathrm{C}_{18}$ silica gel, and preparative HPLC to afford the test compound, OPT. The structure of this isolated compound was determined by a combination of spectroscopic analyses, including IR, ${ }^{1} \mathrm{H}$ and ${ }^{13} \mathrm{C}$ NMR, hydrogen-hydrogen correlation spectroscopy (H-H COSY), heteronuclear multiple quantum coherence (HMQC), heteronuclear multiple bond coherence (HMBC), mass spectroscopic data, and chemical methods. ${ }^{18}$ Based on the spectroscopic analysis, this compound was identified as OPT.

Chemicals and reagents. MG132 was obtained from Fisher Scientific (Chicago, IL, USA). Annexin-V apoptosis kit was obtained from BD Biosciences (San Jose, CA, USA). CHX, NAC CQ. and Tunicamycin were obtained from Sigma (St. Louis, MO, USA). Anti-GRP78, anti-Bim, and anti-PERK antibodies were obtained from Cell Signaling Technology (Danvers, MA, USA). Anti-Ubiquitin, anti- $\beta$-actin, anti-c-Myc and anti-MCL1 antibodies were obtained from Santa Cruz Biotechnology (Santa Cruz, CA, USA).

Cell culture. MCF10A untransformed human mammary epithelial cells, MDAMB231 human breast cancer cells, and HCT116 human colorectal cancer cells were obtained from ATCC. The MEFs used were established by SV40 large T antigen and were described previously. ${ }^{24,25}$ MCF10A cells were maintained in the ATCC-suggested complete growth medium. HCT116 and MDA-MB231 cells were maintained in DMEM supplemented with $10 \%$ fetal bovine serum, mouse bone marrow and MLL-AF9 mouse leukemia cell were maintained in IMEM supplemented with $10 \%$ fetal bovine serum containing $10 \mathrm{ng} / \mathrm{ml} \mathrm{IL-3,10} \mathrm{ng/ml}$ IL- 6 and $100 \mathrm{ng} / \mathrm{ml} \mathrm{SCF}$, and KOPN-1 were maintained in RPMI medium supplemented with $10 \%$ fetal bovine serum. All cells were cultured in a humidified chamber with $5 \% \mathrm{CO}_{2}$ at $37^{\circ} \mathrm{C}$.

Cell viability assay. Cell viability was determined by the MTT assay (SigmaAldrich, St. Louis, MO, USA). MTT was dissolved in PBS at $5 \mathrm{mg} / \mathrm{ml}$ as a stock solution and sterilized using $0.2 \mathrm{~mm}$ filter. MDA-MB231 cells and MCF10A cells were exposed to the indicated concentrations of OPT in a 96-well plate for $48 \mathrm{~h}$ followed by the addition of MTT solution $(0.5 \mathrm{mg} / \mathrm{ml})$ to the cells, and then the solution was incubated at $37^{\circ} \mathrm{C}$ for $4 \mathrm{~h}$. The colored metabolite was dissolved in dimethyl sulfoxide (DMSO). Optical densities were determined on a microplate reader (Molecular Devices, Sunnyvale, CA, USA).

FACS analysis, ROS quantification, and western blot analysis. For the FACS analysis, $2 \times 10^{5}$ cells/well were seeded into 6 -well plates. Samples were prepared based on the instructions provided together with the Annexin-V 
Apoptosis Kit. Briefly, after treatment as indicated in Section, ${ }^{3}$ the adherent and detached cells were collected and washed twice with binding buffer containing $10 \mathrm{mM}$ HEPES, pH 7.4, $140 \mathrm{mM} \mathrm{NaCl}, 2.5 \mathrm{mM} \mathrm{CaCl}$, and then $1 \times 10^{5}$ cells were resuspended in $100 \mu \mathrm{l}$ of binding buffer. In all, $5 \mu \mathrm{l}$ of Annexin V-FITC and $10 \mu \mathrm{l}$ of propidium iodide $(50 \mu \mathrm{g} / \mathrm{ml}$, stocking concentration) were added to the cell suspension. After gently mixing, the cells were incubated for $15 \mathrm{~min}$ at room temperature, and then $400 \mu \mathrm{l}$ of binding buffer was added to get the sample ready. Quantification of cell death was performed using a FACScan (BD Biosciences). Annexin V-positive and/or PI-positive cells were considered as cell death.

Intracellular ROS production was monitored by the permeable fluorescence dye, H2DCFDA. H2DCFDA can readily react with ROS to form the fluorescent product 2,7-dichlorofluorescein (DCF). The intracellular fluorescence intensity of DCF is proportional to the amount of ROS generated by the cells. After the indicated treatment, the cells were incubated with $10 \mu \mathrm{M}$ of H2DCFDA for 30 min and then cells were harvested and resuspended in PBS $\left(10^{6}\right.$ cells $\left./ \mathrm{ml}\right)$. The fluorescence intensity of intracellular DCF (excitation $488 \mathrm{~nm}$, emission $530 \mathrm{~nm}$ ) was measured using FACScan. All the data analyses were performed using the FlowJo analysis software, version 6.0 (Tree Star, Ashland, OR, USA).

Western blot analysis was carried out as described by Li et al. ${ }^{26}$

RNA isolation and RT-PCR. Total RNA was extracted with the RNeasy Mini Kit according to the manufacturer's instructions (Qiagen, Valencia, CA, USA). cDNA was synthesized using M-MLV reverse transcriptase from Promega (Madison, WI, USA). Primer pairs used for RT-PCR are human XBP1, 5'-GGAGTTAAGACAGCGCTTGG-3' (sense) and $5^{\prime}$-ACTGGGTCCAAGTTGT CCAG-3' (antisense); human CHOP, 5'-TGGAAGCCTGGTATGAGGAC-3' (sense) and $5^{\prime}$-TGTGACCTCTGCTGGTTCTG-3' (antisense); human Noxa, $5^{\prime}$-GCTCCAGCAGAGCTGGAAGT-3' (sense) and 5'-GCAGTCAGGTTCCTGA GCAG-3' (antisense); human Bim, $5^{\prime}$-GACTCTGACTCTCGGACTGA-3' (sense) and $5^{\prime}$-GCAGGCTGCAATTGTCTACC-3' (antisense); human GRP78, 5'-TGAA GAGCTCAACATGGATCTGTT- $3^{\prime}$ (sense) and $5^{\prime}$-CTACAGCTTCATCTGGGTT TATGC-3' (antisense); human BCL2, 5'-ATGTGTGTGGAGAGCGTCAA-3' (sense) and $5^{\prime}$-CGTACAGTTCCACAAAGGCA-3' (antisense); human BCL-XL, $5^{\prime}$-CCTTTGCCTAAGGCGGATTT-3' (sense) and $5^{\prime}$-GCTCACTCACTGAGTC TCGT-3' (antisense); human MCL1, 5'-AGAAAGCTGCATCGAACCATT-3' (sense) and $5^{\prime}$-CAGCTCCTACTCCAGCAAC-3' (antisense); human GAPDH, $5^{\prime}$-CTCTGACTTCAACAG CGACAC-3' (sense) and $5^{\prime}$-CATACCAGGAAATGAG CTTGACAA-3' (antisense); mouse XBP1, 5'-ACACGCTTGGGAATGGACAC-3' (sense), mouse GAPDH, 5'-GCACAGTCAAGGCCGAGAAT-3' (sense) and $5^{\prime}$-GCCTTCTCCA TGGTGGTGAA-3' (antisense).

Plasmids and Lentiviral preparation and transduction. Human GRP78 cDNA was subcloned into the lentiviral expression vector pCDH-CMVEF1-puro (System Biosciences, Mountain View, CA, USA). The pLKO.1 lentiviral sh-RNA expression system was used to generate shRNA constructs (sh-GRP78, 5'-CTTGTTGGTGGCTCGACTCGA-3'), (sh-Noxa, 5'-GTAATTATTGACACATT TCTT-3'), (sh-Bim, 5'-CCTTCTGATGTAAGTTCTGTT-3'), and (sh-MCL1, 5'-CGGG ACTGGCTAGTTAAAC-3'). Viral packaging was done according to the protocol described by Li et al..$^{27}$

In vivo xenograft tumor model and xenogen bioluminescence imaging. Female athymic nude mice ( $n=10$ /group, 4-6 weeks of age; Harlan, Indianapolis, IN, USA) were used. The use and care of animals was carried out under the guidelines approved by the Institutional Animal Care and Use Committee. Subconfluent HCT-116-Luc cells were harvested and resuspended in PBS. Before inoculation, cell viability was tested by $0.4 \%$ trypan blue exclusion assay (viable cells $>90 \%$ ). For subcutaneous injection, $\sim 1 \times 10^{6}$ HCT-116-LuC cells in $50 \mu \mathrm{l}$ PBS were injected into both flanks of each mouse for each point. Starting the same day, OPT was intraperitoneally (IP) administered at dose of $15 \mathrm{mg} / \mathrm{kg} / \mathrm{day}$. Control mice were injected with the vehicle. Animal whole body optical imaging was carried out as described previously. ${ }^{28}$ Briefly, animals were subjected to Xenogen IVIS 200 imaging system (Caliper Life Sciences, Hopkinton, MA, USA) for imaging weekly after tumor cell inoculation. D-Luciferin sodium salt (Gold Biotechnology, St. Louis, MO, USA) at $100 \mathrm{mg} / \mathrm{kg}$ body weight in $0.1 \mathrm{ml}$ sterile PBS was administered IP as a substrate before imaging. The acquired pseudo images were collected by superimposing the emitted light over the grayscale photographs of the animal. Quantitative image analysis was performed with Xenogen's Living Image V2.6.1 software (Xenogen Corp, Alameda, CA, USA).
Statistical analysis. Unless otherwise indicated, at least three independent assays were carried out and the values are presented as mean \pm S.D. Statistical significance was assessed by Student's two-tailed $t$ test and $P<0.05$ was considered as statistically significant.

\section{Conflict of Interest}

The authors declare no conflict of interest.

Acknowledgements. We would like to thank Dr. David Ron, Dr. Laurie Glimcher, Dr. Jianjun Chen, Dr. Jinhua Xu, and Dr. Geoffrey Greene for cell lines used in this study. We thank Dr. Arpad Danos for critically reading this manuscript. This work was supported in part by the following grants: NIH/NCCAM AT004418, $\mathrm{NIH} \mathrm{GM074197,} \mathrm{and} \mathrm{NIH/NCl} \mathrm{CA149275.}$

1. Luo J, Solimini NL, Elledge SJ. Principles of cancer therapy: oncogene and non-oncogene addiction. Cell 2009; 136: 823-837.

2. Kim I, Xu W, Reed JC. Cell death and endoplasmic reticulum stress: disease relevance and therapeutic opportunities. Nat Rev Drug Discov 2008; 7: 1013-1030.

3. Walter $P$, Ron $D$. The unfolded protein response: from stress pathway to homeostatic regulation. Science 2011; 334: 1081-1086.

4. Lee AS. The ER chaperone and signaling regulator GRP78/BiP as a monitor of endoplasmic reticulum stress. Methods 2005; 35: 373-381.

5. Lee AS. GRP78 induction in cancer: therapeutic and prognostic implications. Cancer Res 2007; 67: 3496-3499

6. Vembar SS, Brodsky JL. One step at a time: endoplasmic reticulum-associated degradation. Nat Rev Mol Cell Biol 2008; 9: 944-957.

7. Lamark T, Johansen T. Aggrephagy: selective disposal of protein aggregates by macroautophagy. Int J Cell Biol 2012; 2012: 736905.

8. Ogata M, Hino S, Saito A, Morikawa K, Kondo S, Kanemoto $S$ et al. Autophagy is activated for cell survival after endoplasmic reticulum stress. Mol Cell Biol 2006; 26: 9220-9231

9. Tabas I, Ron D. Integrating the mechanisms of apoptosis induced by endoplasmic reticulum stress. Nat Cell Biol 2011; 13: 184-190.

10. Thomenius MJ, Distelhorst CW. Bcl-2 on the endoplasmic reticulum: protecting the mitochondria from a distance. J Cell Sci 2003; 116(Pt 22): 4493-4499.

11. Wei MC, Zong WX, Cheng EH, Lindsten T, Panoutsakopoulou V, Ross AJ et al. Proapoptotic BAX and BAK: a requisite gateway to mitochondrial dysfunction and death. Science 2001; 292: 727-730.

12. Puthalakath H, O'Reilly LA, Gunn P, Lee L, Kelly PN, Huntington ND et al. ER stress triggers apoptosis by activating BH3-only protein Bim. Cell 2007; 129: 1337-1349.

13. Gomez-Bougie P, Wuilleme-Toumi S, Menoret E, Trichet V, Robillard N, Philippe M et al. Noxa up-regulation and $\mathrm{Mcl}-1$ cleavage are associated to apoptosis induction by bortezomib in multiple myeloma. Cancer Res 2007; 67: 5418-5424.

14. Wang $Q$, Mora-Jensen $H$, Weniger MA, Perez-Galan $P$, Wolford $C$, Hai T et al. ERAD inhibitors integrate ER stress with an epigenetic mechanism to activate $\mathrm{BH}$-only protein NOXA in cancer cells. Proc Natl Acad Sci USA 2009; 106: 2200-2205.

15. Zhang L, Lopez H, George NM, Liu X, Pang X, Luo X. Selective involvement of BH3-only proteins and differential targets of Noxa in diverse apoptotic pathways. Cell Death Differ 2011; 18: 864-873.

16. Li C, Li R, Grandis JR, Johnson DE. Bortezomib induces apoptosis via Bim and Bik upregulation and synergizes with cisplatin in the killing of head and neck squamous cell carcinoma cells. Mol Cancer Ther 2008; 7: 1647-1655.

17. Calway T, Du GJ, Wang CZ, Huang WH, Zhao J, Li SP et al. Chemical and pharmacological studies of Oplopanax horridus, a North American botanical. J Nat Med 2012; 66: 249-256.

18. Huang WH, Zhang QW, Wang CZ, Yuan CS, Li SP. Isolation and identification of two new polyynes from a North American ethnic medicinal plant-Oplopanax horridus (Smith) Miq. Molecules 2010; 15: 1089-1096.

19. Wang CZ, Zhang Z, Huang WH, Du GJ, Wen XD, Calway T et al. Identification of potential anticancer compounds from Oplopanax horridus. Phytomedicine 2013; 20: 999-1006.

20. Zhang J, Wang X, Cui W, Wang W, Zhang H, Liu L et al. Visualization of caspase-3-like activity in cells using a genetically encoded fluorescent biosensor activated by protein cleavage. Nat Commun 2013; 4: 2157.

21. Jin HR, Zhao J, Zhang Z, Liao Y, Wang CZ, Huang WH et al. The antitumor natural compound falcarindiol promotes cancer cell death by inducing endoplasmic reticulum stress. Cell Death Dis 2012; 3: e376.

22. Happo L, Strasser A, Cory S. BH3-only proteins in apoptosis at a glance. J Cell Sci 2012; 125(Pt 5): 1081-1087.

23. Nijhawan D, Fang M, Traer E, Zhong Q, Gao W, Du F et al. Elimination of Mcl-1 is required for the initiation of apoptosis following ultraviolet irradiation. Genes Dev 2003; 17 : $1475-1486$. 
24. Harding HP, Zhang $Y$, Ron D. Protein translation and folding are coupled by an endoplasmic-reticulum-resident kinase. Nature 1999; 397: 271-274.

25. Ito D, Walker JR, Thompson CS, Moroz I, Lin W, Veselits ML et al. Characterization of stanniocalcin 2, a novel target of the mammalian unfolded protein response with cytoprotective properties. Mol Cell Biol 2004; 24: 9456-9469.

26. Li B, Zhao J, Wang CZ, Searle J, He TC, Yuan CS et al. Ginsenoside Rh2 induces apoptosis and paraptosis-like cell death in colorectal cancer cells through activation of p53. Cancer Lett 2011; 301: 185-192.

27. Li B, Gordon GM, Du CH, Xu J, Du W. Specific killing of Rb mutant cancer cells by inactivating TSC2. Cancer Cell 2010; 17: 469-480.

28. He BC, Gao JL, Zhang BQ, Luo Q, Shi Q, Kim SH et al. Tetrandrine inhibits Wnt/betacatenin signaling and suppresses tumor growth of human colorectal cancer. $\mathrm{Mol}$ Pharmacol 2011; 79: 211-219.
Cell Death and Disease is an open-access journal published by Nature Publishing Group. This work is licensed under a Creative Commons Attribution-NonCommercialShareAlike 3.0 Unported License. The images or other third party material in this article are included in the article's Creative Commons license, unless indicated otherwise in the credit line; if the material is not included under the Creative Commons license, users will need to obtain permission from the license holder to reproduce the material. To view a copy of this license, visit http://creativecommons.org/ licenses/by-nc-sa/3.0/

Supplementary Information accompanies this paper on Cell Death and Disease website (http://www.nature.com/cddis) 